(C) 2017 IEEE. Personal use of this material is permitted. Permission from IEEE must be obtained for all other uses, in any current or future media, including reprinting/republishing this material for advertising or promotional purposes, creating new collective works, for resale or redistribution to servers or lists, or reuse of any copyrighted component of this work in other works. 


\title{
Low Sidelobe Synthesis of Dipole Arrays by Element Orientation Selection Using Binary Coded Genetic Algorithm
}

\author{
Ming $\mathrm{Li}^{1}$, Yanhui Liu ${ }^{1 *}$, Shu-Lin Chen ${ }^{2}$, Pei-Yuan Qin ${ }^{2}$, Y. Jay Guo ${ }^{2}$ \\ ${ }^{1}$ Department of Electronic Science, Xiamen University, Fujian 361005, China \\ ${ }^{2}$ Global Big Data Technologies Centre, University of Technology Sydney, NSW 2007, Australia \\ * Email: yanhuiliu@xmu.edu.cn;
}

\begin{abstract}
Selecting appropriate element orientations can significantly reduce the sidelobe level of the antenna array. In this paper, a binary coded genetic algorithm (BCGA) which selects the element orientations from specified discrete angles, is proposed to reduce the sidelobe level (SLL) of the array. Compared to the conventional GA, the BCGA is much faster in this application. Synthesis results show the effectiveness and efficiency of the proposed method.
\end{abstract}

Index Terms-antenna array, sidelobe level, element selection, genetic algorithm.

\section{INTRODUCTION}

In the array synthesis, low sidelobe level (SLL) and low cross-polarization level (CPL) are usually preferable [1], [2]. To achieve the desired low sidelobe performance, some optimization algorithms such as the genetic algorithm (GA), differential evolution algorithm (DEA), and particle swarm optimization (PSO), have been proposed [3]-[7]. The main idea of these methods is optimizing the amplitudes and phase of the array's excitation weights to achieve the desired pattern performance. It is very effective but with an additional complexity in the feeding network to implement the synthesized excitation weights. Different from the conventional way of changing the excitation weights, an alternative technique was presented in [8] where the orientation of each element in a dipole array optimized by the GA for reducing the SLL. Lately, such an idea was also applied to reduce the SLL for some other arrays such as the microstrip patch antenna array [9].

In this work, it is shown that the SLL reduction can be implemented through selecting the element orientation from a set of discrete angles. In this case, the binary coded GA can be adopted to find the optimal element orientation with the time cost that is much less than that of the conventional GA used in [8]. Two examples are given for synthesizing the element orientations of linear and planar dipole antenna arrays with SLL and CPL control. The synthesis results show the effectiveness and superiority of the proposed method.

\section{Algorithm Description}

The GA is a heuristic optimization algorithm, which is inspired by the evolution and genetic mechanism of nature. It has been applied to many areas including the synthesis of antenna arrays[1], [3]. In a conventional GA process, variable parameters are coded into chromosomes, and then the fitness function is employed to judge each chromosome's fitness. The chromosomes with good fitness is retained while those with bad fitness is eliminated. For the next step, crossover and mutation are used to increase the diversity of the population. Finally, the fitness of new chromosomes will be calculated and ranked until the best chromosome is generated or the maximum number of iterations is reached.

Different from the conventional GA, a BCGA whose chromosomes or individuals are encoded by a series of binary numbers, is utilized to optimized the orientation of the elements in this work. The selection and copy are implemented by a roulette wheel method.

\section{A. Coding and Fitness Function}

As is known, every digit of the binary chromosome has two forms, 0 and 1 . Hence, for an antenna array, eight elements with different orientations can be expressed by three digits of the chromosome. The fitness function is given through the following equation:

$$
\text { Fit. }=\min (\max (S L L, C P L)),
$$

where the $S L L$ denotes the sidelobe level of the copolarization pattern and the $C P L$ denotes the maximum value of the cross-polarization level. Chromosomes with higher fitness values will be maintained more easily while those with lower fitness values may be in high risk of being eliminated.

\section{B. Selection and Copy}

For a population with $M$ chromosomes, their fitness values could be ranked by descending orders. Their copy probabilities own a relationship with their rank orders as follows:

$$
p_{i}=e(1-e)^{i-1}, \quad(i=1,2, \ldots, M)
$$

where $\mathrm{i}$ represents the rank order of the chromosomes, $e \in$ $(0,1)$ is the copy probability of the first chromosome with the highest fitness value, $p_{i}$ denotes the copy probability of the $i$ th chromosome. It is evident that when $i$ is increased, $p_{i}$ will become smaller and smaller, which obeys the law of survival of the fittest. As also can be seen, every chromosome owns 
TABLE I

CALCULATION OF THE COPY PROBABILITY IN THE ROULETTE WHEEL METHOD

\begin{tabular}{|c|c|c|c|}
\hline$i$ & fitness & $p_{i}=e(1-e)^{i-1}$ & $q_{i}=\sum_{j=1}^{1}\left(p_{j}\right)$ \\
\hline 1 & 10 & 0.40 & 0.40 \\
\hline 2 & 8 & 0.24 & 0.64 \\
\hline 3 & 6 & 0.14 & 0.78 \\
\hline 4 & 2 & 0.09 & 0.87 \\
\hline
\end{tabular}

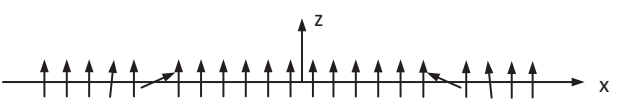

Fig. 1. The optimized elements arrangement

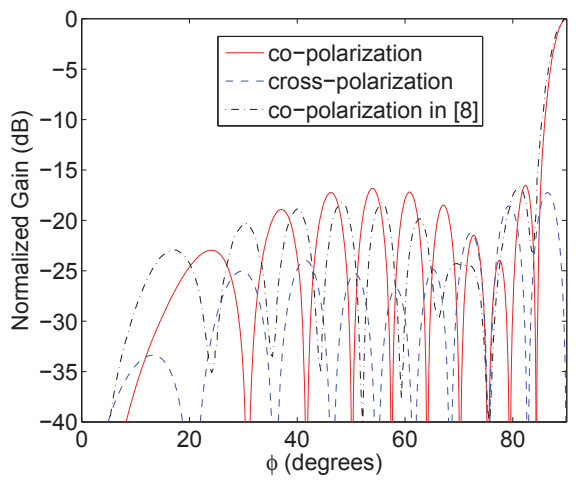

Fig. 2. The co-polarization and cross-polarization pattern of a GA optimized result compared with the co-polarization in [8].

the right of breeding, but the lower the fitness value is, the smaller the breed probability will be.

A roulette wheel method is proposed to deal with the selection in the BCGA. As an example, four individuals are considered in the population. The copy probability is calculated as shown in the Table I, where $q_{i}$ denotes the accumulation of $p_{i}$. When the copy and selection are conducted, four numbers belongs to $(0,0.87)$ are randomly generated at first. And then the selection and copy are implemented with the following rule. That is, when the randomly generated number is located in $(0,0.40]$, the $i=1$ individual will be selected and copied, else if the number is located in $(0.40,0.64]$, the $i=2$ individual will be selected and copied, etc. It can be seen that those chromosomes with larger fitness value could be selected and copied with a larger chance.

\section{Crossover and Mutation}

Crossover and mutation play an important role in the GA since these two steps can keep GA away from earlier convergence and stuck local minimum. During the crossover process, some crossover positions are randomly generated in the parents chromosomes and the exchange of the gene happens between two generated positions to become two new individuals. Similarly, the positions of the mutation are randomly determined and the mutation is carried out through altering one or more digits in every chromosome.

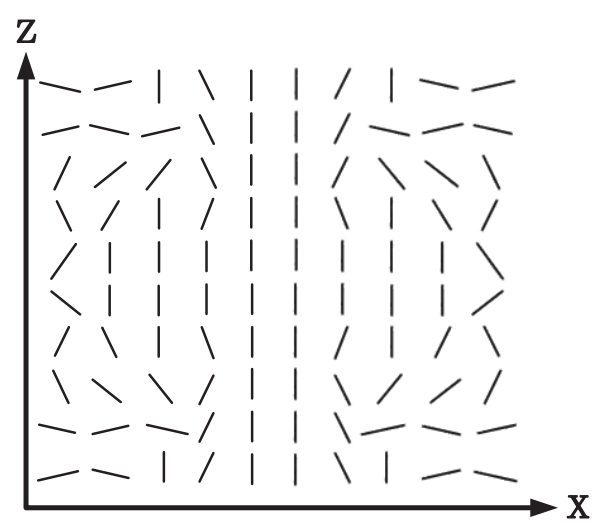

Fig. 3. The elements layout of the optimized planar array.

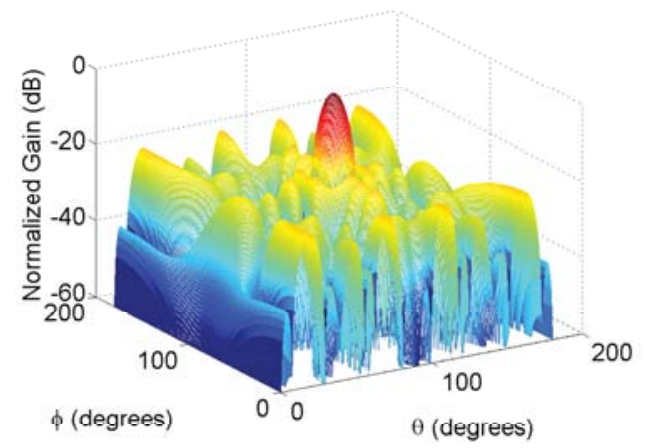

Fig. 4. The pattern of the optimized planar array, with a maximum sidelobe level of $19.33 \mathrm{~dB}$.

\section{OPTIMIZED EXAMPLES}

In order to illustrate the effectiveness of the stated approach, two examples for synthesizing linear and planar dipole arrays are presented in this section.

\section{A. Linear Array}

As the first example, a linear array composed of $\lambda / 2$-spaced dipole elements is considered. The elements are placed along $\mathrm{x}$-axis in $\mathrm{x}-\mathrm{z}$ plane. Hence, the copolarization component is along $\theta$-polarization. For each element, the orientation is chosen from eight discrete direction$\mathrm{s}[0, \pi / 15,2 \pi / 15,3 \pi / 15,4 \pi / 15,5 \pi / 15,6 \pi / 15,7 \pi / 15]$ in the elevation plane. In this case, the population size is chosen as 500 while the maximum number of iterations is set to 50 . The crossover and the mutation probability are $p_{c}=0.6$ and $p_{m}=0.3$, respectively.

Fig. 1 shows the element orientation distribution of the linear array optimized by the proposed BICG. Fig. 2 shows the radiation pattern of this array. As can be seen, the synthesized pattern can achieve a SLL of $16.64 \mathrm{~dB}$ which is very close to the result of $16.7 \mathrm{~dB}$ optimized by the continuous orientation rotation using conventional GA in [8]. Note that in this example, the proposed BICG takes 30.28 seconds on a computer with an Intel Core i5 Processor 2400 (@3.10 GHz) and 4 GB RAM memory, while the conventional GA takes about 16.75 minutes on the same computer. 


\section{B. Planar Array}

In the second example, a $10 \times 10$ planar dipole array is synthesized. The array is arranged in $\mathrm{X}-\mathrm{Z}$ plane with uniform spacing $d=\lambda / 2$. Due to the symmetric structure of this array, only a quarter of elements in the array is optimized. The alternative polarization angles are $-3 \pi / 7,-2 \pi / 7,-\pi / 7,0$, $\pi / 7,2 \pi / 7,3 \pi / 7$, respectively. The crossover and the mutation probability used in this synthesis are also $p_{c}=0.6$ and $p_{m}=0.3$, respectively.

A planar array layout optimized by the BCGA is shown in Fig. 3, and the obtained pattern is shown in Fig. 4. This pattern has a maximum SLL of $19.33 \mathrm{~dB}$ which is roughly equal to $19.4 \mathrm{~dB}$ obtained in [8] for the array of the same size. In this example, the proposed BCGA takes about 1.1 hours while the conventional GA takes 18.63 hours for the optimizing the element rotations in the continuous angle space.

\section{CONCLUSION}

This paper presents a novel element orientation synthesis method which applies the BCGA to efficiently determine the best orientation of every array element from prescribed several discrete angels in terms of the lowest sidelobe level and cross-polarization level. The proposed method is much faster than the method of applying the conventional GA to find the element orientation in continuous angle space, while maintaining a similar pattern performance. This advantage has been validated in the examples of synthesizing the element orientations of both linear and planar dipole arrays. The proposed method can be further generalized to more complicated conformal arrays with different antenna element structures.

\section{REFERENCES}

[1] Yan K K, Lu Y. Sidelobe reduction in array-pattern synthesis using genetic algorithm. IEEE Transactions on Antennas \& Propagation, Vol. 45, No. 7, 1997, pp. 1117-1122.

[2] Ghayoula E. Sidelobe Level Reduction in Linear Array Pattern Synthesis Using Taylor-MUSIC Algorithm for Reliable IEEE 802.11 MIMO Applications. IEEE International Conference on Systems, Man and Cybernetics Smc. 2016.

[3] Laseetha T S J, Sukanesh R. Synthesis of Linear Antenna Array using Genetic Algorithm to Maximize Sidelobe Level Reduction. International Journal of Computer Applications, Vol. 20, No. 7, 2011, pp. 27-33.

[4] Rocha-Alicano C, Covarrubias-Rosales D, Brizuela-Rodriguez C, et al. Differential evolution algorithm applied to sidelobe level reduction on a planar array. AEU - International Journal of Electronics and Communications, Vol. 61, No. 5, 2007, pp. 286-290.

[5] Recioui A. Sidelobe Level Reduction in Linear Array Pattern Synthesis Using Particle Swarm Optimization. Journal of Optimization Theory \& Applications, Vol. 153, No. 153, 2012, pp. 497-512.

[6] Zaharis Z D, Skeberis C, Xenos T D. Improved Antenna Array Adaptive Beamforming with Low Side Lobe Level Using a Novel Adaptive Invasive Weed Optimization Method. Progress in Electromagnetics Research,Vol. 14, No. 8, 2012, pp. 137-150.

[7] Quevedo-Teruel O, Rajo-Iglesias E. Ant Colony Optimization in Thinned Array Synthesis With Minimum Sidelobe Level. IEEE Antennas \& Wireless Propagation Letters, Vol. 5, No. 1, 2007, pp. 349-352.

[8] Haupt R L, Aten D W, Low Sidelobe Arrays via Dipole Rotation, IEEE Transactions on Antennas \& Propagation, Vol. 57, No. 5, 2009, pp. 15751579 .

[9] Zhang F, Zhang F S, Lin C, et al. Pattern Synthesis for Planar Array Based on Elements Rotation. Progress in Electromagnetics Research Letters, Vol. 11, 2009, pp. 55-64. 